\title{
Factores predictores de uso de drogas ilegales en personas atendidas en una sala de emergencia.
}

\author{
Predictor factors of illegal drug use in subjects attended in an emergency room. \\ Enrique Ramírez ${ }^{1}$, José Quintana ${ }^{1}$, Hans Martel$^{1}$, Javier Ponce ${ }^{1}$, Johann Vega-Dienstmaier ${ }^{2}$, \\ Carla Gallo ${ }^{3}$, Inés Bustamante ${ }^{4}$, Carlos Ordóñez ${ }^{5}$, Guido Mazzotti ${ }^{6 \dagger}$, Fabián Fiestas ${ }^{7}$
}

\section{RESUMEN}

Objetivo: Valorar el consumo reciente de alcohol y otras variables como predictores del uso reciente y consumo de vida de alguna droga ilegal, entre personas atendidas en una sala de emergencia (SE) de un hospital de Lima. Materiales y Métodos: Este estudio es un análisis secundario de datos de un trabajo previo observacional de corte transversal realizado por Mazzotti et al. (2005), cuya muestra estuvo constituida por 371 pacientes, que involucraron a todos los que fueron atendidos en la SE (Medicina y Cirugía) del Hospital Nacional Cayetano Heredia durante siete días completos de enero del año 2005. Se aplicó la encuesta del Sistema Interamericano de Datos sobre el Uso de Drogas (SIDUC) desarrollada por la Comisión Interamericana para el Control del Abuso de Drogas (CICAD) para uso reciente de sustancias psicoactivas. Resultados: El consumo reciente de alguna droga ilegal estuvo fuertemente asociado con el consumo reciente de alcohol (OR=32,21; IC 95\%=9,0-114,5; $\mathrm{p}<0,001)$ y el sexo masculino (OR=4,52; IC 95\%=1,2-15,8; $p=0,018$ ), lo cual se mantuvo aún después de ajustar por posibles variables de confusión. Asimismo, estas variables (consumo reciente de alcohol y sexo) mostraron asociación significativa con el consumo de vida de marihuana y cocaína. Conclusiones: El consumo reciente de alcohol y el sexo predicen fuertemente tanto uso reciente como uso de vida de alguna droga ilegal en el contexto de una SE, por lo que estas características pueden ser utilizadas para estrategias de tamizaje dirigidas a la intervención y manejo de problemas de drogas a nivel de servicios hospitalarios. (Rev Neuropsiquiatr 2010;73:136-146).

PALABRAS CLAVE: Drogas ilegales, alcohol, sala de emergencia, consumo reciente, consumo de vida.

\footnotetext{
1 Médico. Universidad Peruana Cayetano Heredia. Lima, Perú.

2 Psiquiatra. Universidad Peruana Cayetano Heredia, Hospital Nacional Cayetano Heredia. Lima, Perú.

Biólogo. Laboratorios de Investigación y Desarrollo, Universidad Peruana Cayetano Heredia. Lima, Perú.

4 Psicóloga salubrista, Universidad Peruana Cayetano Heredia. Lima, Perú.

5 Psiquiatra. Jefe del Departamento de Adicciones, Hospital Hermilio Valdizán. Lima, Perú.

$6 \quad$ Psiquiatra. Universidad Peruana Cayetano Heredia. Lima, Perú.

7 Médico Epidemiólogo. Universidad Peruana Cayetano Heredia. Lima, Perú.

† In Memoriam.
} 
Factores predictores de uso de drogas ilegales en personas atendidas en una sala de emergencia.

\section{SUMMARY}

Objective: To assess recent alcohol consumption and other variables as predictors of recent use and lifetime use of illegal drugs among people visiting an emergency room in a hospital in Lima. Materials and Methods: This study is a secondary analysis of data from a previous study by Mazzotti et al. (2005). It had a cross-sectional observational design, and the sample consisted of 371 patients, involving all patients who were treated in the emergency room (Medicine and Surgery) of the Hospital Nacional Cayetano Heredia for seven full days in January 2005. The InterAmerican System of Uniform Drug-Use Data (SIDUC) survey developed by the Inter-American Drug Abuse Control Commission (CICAD) was applied for recent use of psychoactive substances in emergency rooms. Results: We found strong association between recent use of any illegal drugs and both having recent alcohol use (OR=32.21; $95 \% \mathrm{CI}=9.0-114.5 ; \mathrm{p}<0.001)$ and being a male $(\mathrm{OR}=4.52 ; 95 \% \mathrm{CI}=1.2-15.8 ; \mathrm{p}=0.018)$. These associations were kept even after adjustment for meaningful covariates. In addition, these characteristics (recent alcohol consumption and male) showed significant association with lifetime use of marijuana and cocaine. Conclusions: Recent alcohol use and sex were strong predictors of both recent and lifetime use of illegal drugs. Therefore, these individual-level features can be used in hospital-based screening strategies aimed to identify drug-related problems and provide timely help. (Rev Neuropsiquiatr 2010;73:136-146).

KEYWORDS: illegal drugs, alcohol, emergency room, recent use, lifetime use.

\section{INTRODUCCIÓN}

Los trastornos por consumo de alcohol y drogas ilegales siguen siendo uno de los principales problemas de salud pública en el Perú y el mundo. En nuestro país, la marihuana es la sustancia ilegal de mayor consumo, con un $6,3 \%$ de incidencia acumulada en la población general, seguida de la cocaína (3,9\%) (1). Además, también encontramos altos niveles de trastornos por uso de alcohol, siendo los más altos en la sierra y la selva en donde la incidencia acumulada de estos trastornos es de $10,5 \%$ y $8,8 \%$, respectivamente (2). Del mismo modo, el Informe sobre la Salud en el Mundo del 2002 (3) señaló que el 8,9\% de la carga total de morbilidad se debe al consumo de sustancias psicoactivas.

El uso de alcohol y drogas ilegales está asociado a conducta violenta, accidentes y otros problemas de salud. La fuerte relación entre el uso de sustancias psicoactivas y las lesiones (4-8) causa que las personas con uso de alcohol y consumo de drogas ilegales sean más propensas a usar una sala de emergencia (SE) (9). En el año 2007, según el sistema DAWN (Drug Abuse Warning Network) se encontró que en EEUU, de 116 millones de visitas a SE, 1,9 millones estuvieron asociadas al uso o abuso de sustancias, y de éstas $28 \%$ al consumo de drogas ilegales (10). Asimismo, en un estudio realizado en Costa Rica en tres diferentes SE (11), se encontró que aproximadamente el 50\% de las personas que acuden a emergencia con algún evento traumático presenta consumo reciente (6 horas) de sustancias psicoactivas. En el Perú, un estudio en siete SE de Lima Metropolitana reveló que el 58\% de las atenciones de lesiones por accidentes y agresión estuvieron asociadas al consumo de alcohol (12).

Aunque la relación entre lesiones y uso de alcohol en el contexto de una SE ha sido bastante estudiada, la referida a lesiones y uso de drogas ilegales ha sido menos investigada. Entre los estudios más representativos en esa línea se encuentra uno realizado en Holanda, donde se encontró que el consumo de drogas ilegales (uso de marihuana, cocaína, anfetaminas, éxtasis, heroína, alucinógenos, ácido hidroxibutírico y metadona en las últimas 24 horas) eran frecuentes en SE y que su presencia se asociaba al sexo, la edad, el uso frecuente de alcohol y la región de ese país (13). En el Perú, Mazzotti et al. encontraron que 3,6\% de personas atendidas en los servicios de medicina, cirugía y gínecoobstetricia reportaron haber consumido alguna droga ilegal en las 6 horas previas a su arribo a la SE de un hospital público de Lima Metropolitana (14).

Una de las razones por las que es importante estudiar en qué medida el consumo de sustancias está presente en servicios hospitalarios, es porque éstos podrían representar un escenario excepcional para identificar personas con uso problemático de sustancias y ofrecerles la primera oportunidad de ayuda. Algunas experiencias en este sentido son alentadoras. Por ejemplo, el proyecto ASSERT (Alcohol and Substance 
Abuse Services Education and Referral to Treatment), que es un programa integrado a SE, en que pacientes con uso problemático de alcohol y drogas son tamizados, intervenidos mediante sesiones breves de consejería y finalmente derivados a un centro especializado con resultados prometedores (15).

Por su parte, para el Perú, nuestro grupo encontró que se justifica el tamizaje direccionado, usando factores predictores de uso problemático crónico de alcohol, recomendando que se implemente en SE iniciativas costo-efectivas para lograr que más pacientes con estos problemas sean identificados y manejados (16). En la misma línea, el presente trabajo tiene un objetivo similar para drogas ilegales e inhalantes, usando los datos del mismo estudio.

Específicamente, el presente reporte intenta valorar qué características pueden predecir la presencia de uso reciente y de vida de drogas ilegales (que incluyen, marihuana, cocaína/crack/PBC) e inhalantes. Las características predictoras seleccionadas para estudio son aquellas que puedan ser rápida y fácilmente identificables en el contexto de una SE, como el sexo, la edad, el servicio de emergencia en que fue atendido el paciente (i.e., cirugía o medicina), la presencia de lesiones y el consumo reciente de alcohol (i.e., en las 6 horas previas a la atención en la SE). El objetivo ulterior de este estudio es identificar factores marcadores que podrían ayudar a la detección de individuos con uso problemático de estas sustancias, sirviendo como base para la instauración de programas de manejo temprano de personas con problemas de uso de sustancias ilegales en SE.

\section{MATERIAL Y MÉTODOS}

Este estudio es observacional de tipo transversal, y es resultado de un análisis secundario de la base de datos del trabajo "Consumo de alcohol y otras sustancias psicoactivas en pacientes atendidos en un servicio de emergencia en Lima-Perú” (14). La población de estudio fueron todos los pacientes mayores de 14 años atendidos en los tópicos de cirugía o medicina de la SE de un hospital público de la ciudad de Lima, en el lapso de usa semana completa en enero del 2005. Se aplicó una encuesta clínico-epidemiológica, compuesta por el SIDUC (Sistema de Datos Uniformes sobre Consumo de Drogas) y el AUDIT (Alcohol Use Disorder Identification Test). En el estudio base de Mazzotti et al. (14) se describen prevalencias de consumo de alcohol y otras sustancias psicoactivas, mientras que en el presente trabajo se busca identificar variables predictoras del consumo de alguna droga ilegal (sexo, uso reciente de alcohol, lesiones, etc.) con el objetivo de obtener marcadores de tamizaje para identificar este problema en el contexto de una SE. El tipo de muestreo fue no probabilístico y por conveniencia. Los criterios de inclusión comprendieron a hombres y mujeres mayores de 14 años que solicitaron atención en la SE del Hospital Nacional Cayetano Heredia y que autorizaron su participación en el estudio. En el caso de menores de edad, se solicitó la autorización también de los padres, tutores o persona responsable. Fueron excluidas todas aquellas personas que no consintieron su participación y las que requirieron manejo clínico en las unidades de shock/trauma. Aunque el proyecto primario incluyó a las mujeres atendidas en el tópico de gíneco-obstetricia, para el presente reporte éstas son excluidas del análisis ya que un gran porcentaje de ellas fueron mujeres en trabajo de parto y pocas pacientes reportaron haber consumido recientemente alcohol o alguna droga ilegal.

Las variables operacionales fueron consumo reciente de alcohol, de alguna droga ilegal (marihuana, clorhidrato de cocaína, pasta básica de cocaína [PBC] o crack) o inhalantes en las 6 horas previas al arribo a la sala de emergencia, consumo alguna vez de marihuana, consumo alguna vez de cocaína (clorhidrato de cocaína o PBC), edad, sexo, tópico de emergencia al que llegó el paciente (medicina o cirugía), lesión (existencia de daño físico) y uso problemático de alcohol (AUDIT = 8 ó más).

El análisis estadístico siguió la iteración de pasos explorar-estimar-explorar. El primer paso, incluye la fase descriptiva, en que para las variables categóricas se calcularon porcentajes con sus respectivos intervalos de confianza al 95\%, y para las variables continuas, como la edad, se realizaron análisis de tendencia central (media, mediana, desviación estándar y percentiles), los cuales ayudaron a establecer categorías de grupos etáreos presentados en la tabla 1. El segundo paso, de estimación de asociaciones, incluyó análisis de asociación bivariada a través de Chi cuadrado o test de Fisher según fue apropiado para el tamaño de las celdas para cada variable participante en las tablas de contingencia (si en alguna celda el tamaño fue de 5 ó menos sujetos, se usó el test de Fisher para valorar asociación entre dos variables categóricas).

El análisis para valorar efectos de predicción independiente (por ejemplo para la edad, sexo, 
especialidad del servicio de atención, presencia de daño físico y uso reciente de alcohol) se realizó con regresiones logísticas multivariadas. Todas las asociaciones fueron valoradas en el contexto de un alfa menor al 5\%. El tercer paso, incluyó la exploración de posibles efectos de modificación entre variables independientes y posibles violaciones a la bondad de ajuste de los modelos de regresión.

\section{RESULTADOS}

El presente estudio se basó en el análisis de un total de 371 participantes. En la tabla 1, se puede apreciar que 55\% de los participantes del estudio fueron varones $(\mathrm{n}=200) ;$ en cuanto al grupo etáreo el rango de 19 a 25 años fue el más numeroso con 23\% (n=86). El 61\% $(\mathrm{n}=224)$ de los participantes acudieron al tópico de

Tabla 1: Distribución de los participantes según las variables de estudio

\begin{tabular}{|c|c|c|}
\hline & $\mathbf{n}$ & $\%$ \\
\hline \multicolumn{3}{|l|}{ Sexo } \\
\hline Femenino & 159 & 44,29 \\
\hline Masculino & 200 & 55,71 \\
\hline \multicolumn{3}{|l|}{ Edad } \\
\hline De 14 a 18 & 41 & 11,05 \\
\hline De 19 a 25 & 86 & 23,18 \\
\hline De 26 a 34 & 83 & 22,37 \\
\hline De 35 a 44 & 55 & 14,82 \\
\hline De 45 a 64 & 72 & 19,41 \\
\hline 65 ó mayor & 34 & 9,16 \\
\hline \multicolumn{3}{|l|}{ Tópico del Servicio de Emergencia } \\
\hline Medicina & 224 & 61,04 \\
\hline Cirugía & 143 & 38,96 \\
\hline \multicolumn{3}{|l|}{ Lesión (existencia de daño físico) } \\
\hline Sí & 123 & 33,51 \\
\hline No & 244 & 66,49 \\
\hline \multicolumn{3}{|l|}{ Alcohol en las últimas 6 horas } \\
\hline Sí & 66 & 17,79 \\
\hline No & 305 & 82,21 \\
\hline \multicolumn{3}{|l|}{ Consumo de drogas ilegales } \\
\hline Por lo menos una droga ilegal & 19 & 5,12 \\
\hline Ninguna & 352 & 94,88 \\
\hline \multicolumn{3}{|l|}{ Consumo de cualquier SPA } \\
\hline Por lo menos un SPA & 89 & 23,99 \\
\hline Ninguna & 282 & 76,01 \\
\hline \multicolumn{3}{|l|}{ Consumo de vida de marihuana } \\
\hline Sí & 50 & 14,25 \\
\hline No & 301 & 85,75 \\
\hline \multicolumn{3}{|l|}{ Consumo de vida de cocaína } \\
\hline Sí & 55 & 15,66 \\
\hline No & 296 & 84,33 \\
\hline
\end{tabular}




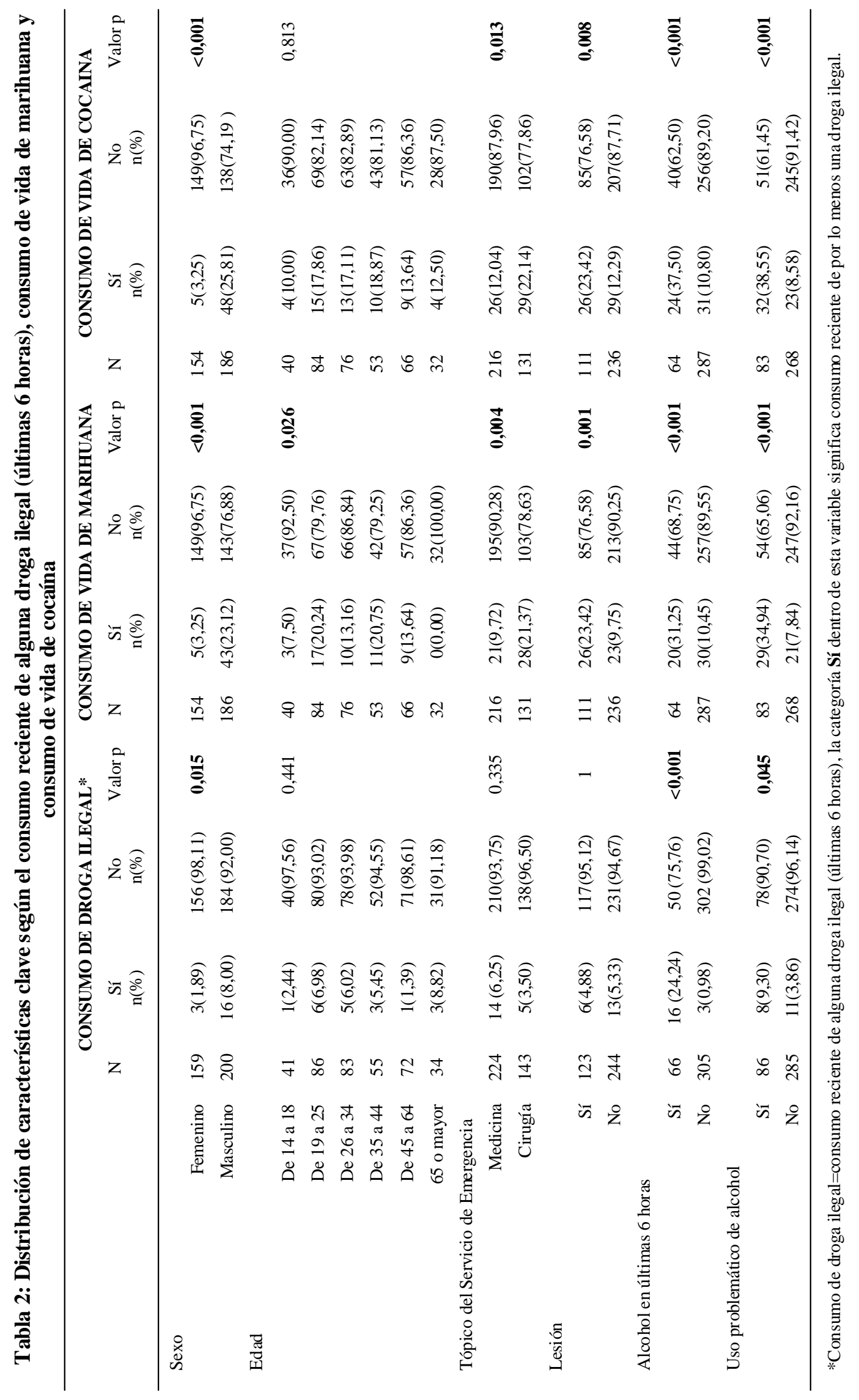


Factores predictores de uso de drogas ilegales en personas atendidas en una sala de emergencia.

medicina y el 39\% $(n=143)$ al de cirugía. El 33\% ( $n=123)$ presentó alguna lesión. Respecto al uso reciente (en las 6 horas previas al arribo a la SE) de sustancias psicoactivas, el $17 \%(n=66)$ presentó consumo reciente de alcohol y el 5\% $(n=19)$ presentó consumo reciente de alguna droga ilegal (que incluye marihuana, clorhidrato de cocaína, PBC, crack o inhalantes). En cuanto al uso de vida, el 14\% ( $\mathrm{n=50)}$ había usado marihuana alguna vez, y el 15\% $(n=55)$ cocaína.

En el análisis bivariado entre el consumo reciente de alguna droga ilegal y las variables de interés (Tabla 2), se observa que los varones tienen más probabilidad que las mujeres de haber consumido por lo menos una droga ilegal en las últimas 6 horas $(\mathrm{p}<0,015)$. Por otra parte, se encuentra una fuerte asociación entre consumo reciente de por lo menos una droga ilegal y consumo reciente de alcohol $(\mathrm{p}<0,001)$, ocurriendo que el $24 \%$ de los que presentaron consumo reciente de alcohol también tuvieron consumo reciente de por lo menos una de las drogas ilegales. Asimismo, las personas que presentaron consumo reciente de alguna droga ilegal tienen más probabilidad de presentar uso problemático de alcohol, sin embargo la asociación fue marginal desde el punto de vista estadístico $(p=0,045)$. Las variables edad, tópico de emergencia y presencia de lesión no tuvieron asociación estadísticamente significativa con respecto al consumo reciente de alguna droga ilegal.

La tabla 2 muestra además los resultados del análisis bivariado del consumo de vida tanto de marihuana y de cocaína con respecto a las variables de estudio. Se encontró asociación estadísticamente significativa entre el consumo de vida de marihuana y cocaína con el sexo, ocurriendo que el $23 \%$ y $25 \%$ de los varones presentaron consumo de vida de marihuana y cocaína, respectivamente, lo cual fue mayor que los correspondientes porcentajes estimados para las mujeres ( $<<0,001$ para ambos casos, marihuana y cocaína). También se encontró que entre aquellos con consumo de alcohol en las últimas 6 horas, el 31\% reportó consumo de vida de marihuana y 37,5\% de cocaína, lo cual significó que el consumo reciente de alcohol se asocia al uso de vida tanto de marihuana como de cocaína ( $\mathrm{p}<0,001$, para ambos casos).

Asimismo, se encontró que el 35\% y 39\% de los participantes con consumo de vida de marihuana y cocaína tuvieron uso problemático de alcohol en el último año $(\mathrm{p}<0,001$ para la asociacion entre el uso problematico de alcohol y el consumo de vida de marihuana y el de cocaina). Con respecto al servicio al que acudieron los participantes, se encontró que aproximadamente el $21 \%$ y $22 \%$ de los participantes que acudieron al servicio de cirugía tuvieron consumo de vida de marihuana y cocaína respectivamente, situación que se reflejó también en una asociación estadísticamente significativa con la presencia de lesión ( $\mathrm{p}=0,004$ para el caso de la marihuana, y p=0,013 para el caso de la cocaína). La variable edad no tuvo una asociación estadísticamente significativa con el consumo de vida de cocaína, mas sí con el consumo de vida de marihuana $(p=0,026)$, encontrándose que aproximadamente un $20 \%$ de personas de 19 a 25 años y un $21 \%$ de personas de 35 a 44 años tuvieron consumo de marihuana al menos una vez en lo que va de sus vidas.

La tabla 3 muestra los Odds Ratio (OR), intervalos de confianza al 95\% y valores p, de la asociación tanto cruda como ajustada entre consumo reciente de alguna droga ilegal y las variables de interés. El hallazgo más resaltante es que los odds de tener un consumo reciente de alguna droga ilegal para los que presentan consumo reciente de alcohol (en las últimas 6 horas) es 32,2 veces los odds de aquellos sin consumo reciente de alcohol (IC al 95\%: 9-114, $\mathrm{p}<0,001$ ). Asimismo, con respecto al sexo, los odds de tener consumo reciente de alguna droga ilegal para los varones son 4,5 veces los odds de tener dicho consumo en las mujeres.

Luego, en el modelo que mantiene constante el sexo, la edad y el tópico de atención en la sala de emergencia, la asociación entre el consumo reciente de alguna droga ilegal y el consumo reciente de alcohol persistió $(p<0,001)$. Asimismo, la asociación entre consumo reciente de alguna droga ilegal y el sexo se mantuvo, en el modelo ajustado. Por otra parte, no se puede concluir sobre la asociación entre el consumo reciente de alguna droga ilegal y el uso problemático de alcohol, pues el valor $\mathrm{p}(\mathrm{p}=0,052)$ se encuentra en el límite del valor alfa establecido para hacer juicios estadísticos (i.e., 0,05).

En la tabla 4 se muestran los OR, intervalos de confianza al 95\% y los valores de p de la asociación no ajustada y ajustada entre consumo de vida de marihuana, consumo de vida de cocaína con respecto a las variables de interés. Se observa que los odds de consumo de vida de marihuana y cocaína en los hombres son 8,9 y 10,3 veces, respectivamente, los odds de tener dicho consumo entre las mujeres. Asimismo, los odds de consumo de vida de marihuana y cocaína en personas con uso de alcohol en las últimas 6 horas son 3,8 y 4,9 
Tabla 3: Asociación entre características clave de individuos atendidos en una sala de emergencia con el consumo reciente de alguna droga ilegal

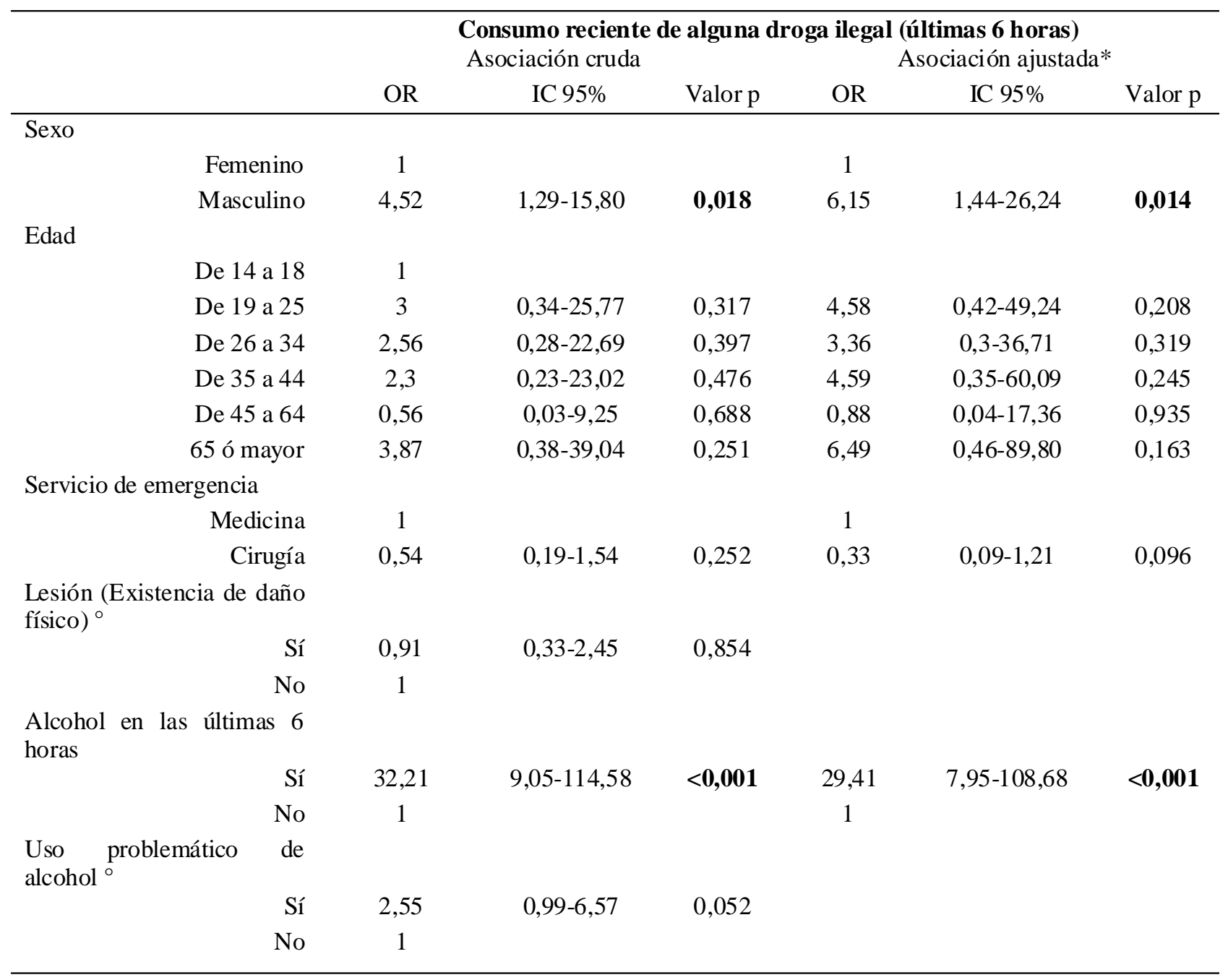

\footnotetext{
${ }^{\circ}$ La variable correspondiente a Lesión está fuertemente asociada a la variable correspondiente al tópico del servicio de emergencia donde fue atendido el paciente por lo que sólo una de ellas tomada para el análisis ajustado; lo mismo ocurre con las variables alcohol en las últimas 6 horas y uso problemático de alcohol. *La estimación de los ORs se realizó en un modelo que incluyó las variables sexo, edad, servicio de emergencia, alcohol en las últimas 6 horas, simultáneamente.
}

veces, respectivamente, los odds de tener dicho consumo entre los que no presentaron uso de alcohol en las últimas 6 horas. Similarmente, los odds de consumo de vida de marihuana y cocaína en las personas con uso problemático de alcohol son 6,3 y 6,8 veces respectivamente los odds de tener dicho consumo entre los que no tienen uso problemático de alcohol, todas estas asociaciones sostenidas con un $\mathrm{p}$ $<0,001$. También se encuentra que los odds de consumo de vida de marihuana y cocaína en el servicio de cirugía son 2,5 y 2,1 veces, respectivamente, los odds de tener dicho consumo entre los atendidos en el servicio de medicina. Asimismo, los odds de consumo de vida de marihuana y cocaína con presencia de lesión son 2,8 y 2,2 veces, respectivamente, los odds de tener dicho consumo entre aquellos que no presentan lesión, sin embargo estas asociaciones tuvieron un valor p $>0,05$ en el modelo ajustado. En lo que respecta a la variable edad, la asociación tuvo un valor $\mathrm{p}>0,05$ para todos los casos. 
Tabla 4: Asociación entre características clave de individuos atendidos en una sala de emergencia con el consumo de vida de marihuana y consumo de vida de cocaína.

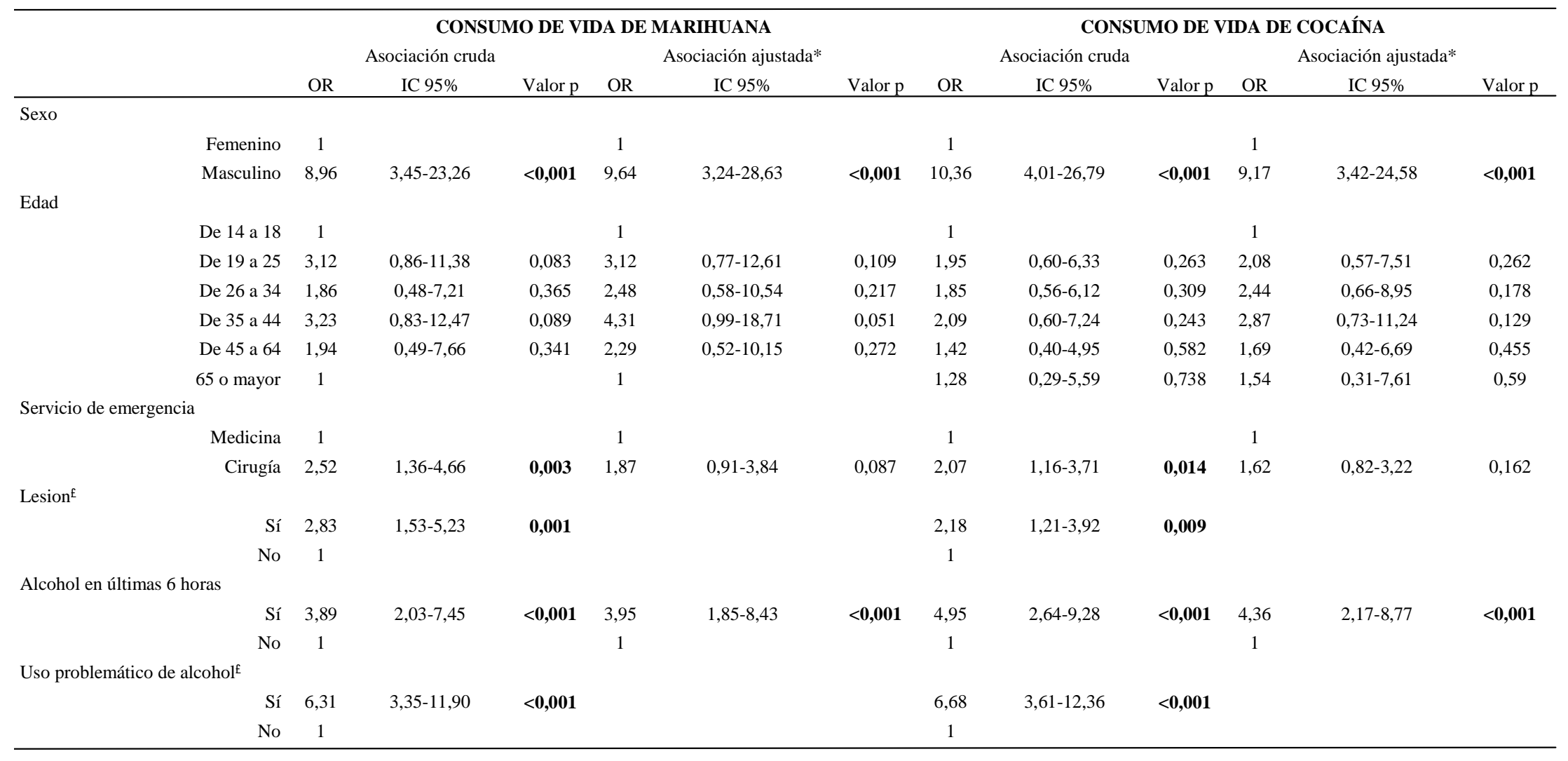

${ }^{£}$ La variable correspondiente a Lesión está fuertemente asociado a la variable correspondiente al servicio donde fue atendido el paciente por lo que sólo una de ellas tomada para el análisis ajustado; lo mismo ocurre con las variables alcohol en las últimas 6 horas y uso problemático de alcohol.

*Significa que la estimación de los ORs se realizó en un modelo que incluyó las variables sexo, edad, tópico del servicio de emergencia, alcohol en las últimas 6 horas, simultáneamente 


\section{DISCUSIÓN}

El principal hallazgo del presente estudio es que de las personas que acuden a una SE, el ser varón y presentar consumo reciente de alcohol (6 horas previas) tienen un fuerte efecto predictor del consumo reciente y de vida de alguna droga ilegal, siendo esto independiente de la edad, presencia de daño físico/lesión o del tópico de la sala de emergencia a la que acuden.

Estos resultados sugieren que el sexo y el consumo reciente de alcohol pueden usarse para identificar personas con consumo, tanto reciente como de vida, de drogas ilegales entre aquellos que son atendidos en una SE. Aunque el presente estudio tiene la limitación de que no valoró específicamente criterios de abuso o dependencia a drogas ilegales, es probable que el uso reciente de estas sustancias psicoactivas pueda ser un marcador de persistencia de uso y por ende ser un marcador de existencia de abuso o dependencia. Futuros estudios que evalúen si, efectivamente, el sexo y el uso reciente de alcohol son también predictores de abuso o dependencia a drogas ilegales en el contexto de una sala de emergencia deben realizarse, como se ha visto que es el caso para identificar uso problemático de alcohol (16).

De ser el caso que estas variables -cuyo registro no significa una sobrecarga de trabajo para los profesionales de salud en las salas de emergenciafueran también predictoras de abuso o dependencia a drogas ilegales, entonces podrían ser consideradas en los protocolos de atención como herramienta para identificar estos problemas en las salas de emergencia, ayudando a reconocer a quienes se debería aplicar instrumentos de tamizaje más extensos y específicos. Ello significaría entonces un mecanismo costo-efectivo para identificar y brindar a los usuarios de drogas ilegales una breve consejería sobre este problema, y posiblemente una derivación a servicios especializados. El hecho de que los procesos de identificación de estas personas con uso, quizá problemático, de drogas ilegales sea relativamente sencillo y de bajo costo, más el hecho de que existen experiencias exitosas para manejar dicho problema, hace que recomendemos la implementación de medidas tamizaje de estos problemas en las SE. Ello facilitaría el acceso a un cuidado médico de personas con uso problemático de estas sustancias, las cuales actualmente pasan desapercibidas y son dejadas a una suerte incierta habiendo tenido la oportunidad de ser detectadas tempranamente durante su atención en un establecimiento médico y manejadas apropiadamente.

En ese sentido, es alentador conocer de la experiencia de la implementación de sistemas similares en países desarrollados, como el programa ASSERT (Alcohol and Substance Abuse Services Education and Referral to Treatment), en EE.UU. Este programa significó la aplicación de medidas basadas en SE para detectar, aplicar intervenciones breves, y referir cuando corresponde, a los pacientes a un programa para tratamiento de uso problemático de alcohol y otras drogas, $y$ ha mostrado ser exitoso en cuanto a la alta tasa de pacientes que se acoplaban a los programas de tratamiento (15). Otra experiencia similar es la del programa SBIRT (Screening, Brief Interventions, Referral to Treatment), en el que se ha mostrado que a los 6 meses de haberse implementado en los centros de salud hubo reducción significativa de los niveles de consumo de drogas ilegales y del uso no saludable de alcohol entre aquellos que reportaron algún tipo de consumo (17).

Además del hecho de que no se evaluó específicamente la presencia de abuso o dependencia a drogas ilegales, otras limitaciones del estudio pueden ser mencionadas. La primera tiene que ver con la amenaza a la validez externa de nuestros hallazgos debido a que se incluyó la SE de un solo hospital por motivos de presupuesto. Sin embargo, el hospital donde se realizó el estudio tiene bajo su jurisdicción una amplia área geográfica con características socio-demográficas muy representativas de Lima Metropolitana; ello permitiría extrapolar los hallazgos a otras regiones populosas de la misma ciudad. No obstante, es recomendable replicar este estudio en otros hospitales de Lima y del resto del país, ya que las características socioeconómicas en otras zonas del Perú pueden hacer variar la fuerza de predicción de las variables estudiadas (por ejemplo, ciudades grandes vs. pequeñas, ambientes urbanos vs. rurales), con el correspondiente impacto en su costo-efectividad al ser usadas en el proceso de identificación de posibles casos de uso problemático de drogas ilegales, como es sugerido también por la literatura (13). Una segunda limitación, que debe ser tomada en cuenta para futuros estudios, es que en este trabajo no se contó, por motivos de presupuesto, con pruebas toxicológicas de laboratorio en sangre u orina suficientes como para hacer análisis de validez para detectar uso reciente de las drogas en los participantes, lo cual hubiera significado un diagnóstico más objetivo, y probablemente la captación de más pacientes con 
Factores predictores de uso de drogas ilegales en personas atendidas en una sala de emergencia.

uso reciente de drogas ilegales, que pudieron haber negado su uso durante la entrevista (14).

Otra limitación es que existe la posibilidad de sesgo de reporte ligado al sexo. Ello significa que, los patrones de conducta que se desprenden de los roles socioculturalmente esperados de las mujeres, podrían hacerlas sentir obligadas a ocultar su uso por temor a crear juicios de valor sobre ellas. Sin embargo, se espera que este sesgo sea mínimo, ya que en todo momento se guardó la confidencialidad de los sujetos, y además los entrevistadores fueron capacitados para poder llevar una entrevista adecuada.

Por otra parte, otra limitación del estudio fue que el tamaño de muestra fue limitado, pues se trata de un análisis secundario de una base de datos pre-existente, lo que llevó a que algunos resultados presenten intervalos de confianza poco precisos. Sin embargo, los OR sobrepasan el valor de 1 y los valores p para las variables principales fueron bastante robustos, a niveles incluso menores a 0,001. Futuros estudios ad hoc deberán incrementar el tamaño muestral, especialmente para poder realizar una evaluación individual de cada droga ilegal.

Entre las fortalezas del estudio, se encuentra que hasta donde conocemos, éste sería el primer estudio en nuestro medio que intenta identificar características para potenciar la costo-efectividad de posibles medidas para tamizar personas con potenciales problemas relacionados al uso de drogas ilegales en el contexto de SE. Además, se trató de un estudio de tipo epidemiológico en un hospital público representativo de Lima Metropolitana, por lo que puede ser extrapolado a otros hospitales de características similares.

Tras lo antes expuesto, se puede concluir que el ser varón y tener uso reciente de alcohol son variables predictivas tanto de uso reciente de alguna droga ilegal, como también de uso de vida de marihuana y/o cocaína, en el contexto de una SE. Por ello, estas características pueden ayudar a seleccionar a aquellas personas que ameritan ser tamizadas por problemas de uso de sustancias, aunque ello permanece por ser estudiado específicamente en muestras más grandes, en un mayor número de hospitales y con instrumentos ad hoc para abuso o dependencia de drogas. El tamizar por abuso o dependencia a drogas permitirá que personas que necesitan ayuda por esos problemas reciban el apoyo apropiado tempranamente, lo que ulteriormente llevará a una reducción de la carga de enfermedad generada por estas sustancias.

\section{Correspondencia}

Fabian Fiestas, M.D., Ph.D. (c)

Unidad de Neurobiología Molecular,

Laboratorios de Investigación y Desarrollo,

Universidad Peruana Cayetano Heredia.

Av. Honorio Delgado 430, San Martín de Porres, Lima, Perú.

Teléfono: (+511) 985-765-743

Correo electronico: ffiestas@epi.msu.edu

\section{REFERENCIAS BIBLIOGRÁFICAS}

1. Comisión Nacional para el Desarrollo y Vida sin Drogas - DEVIDA. II Encuesta Nacional sobre Prevención y Consumo de Drogas 2002. Lima, Perú: DEVIDA; 2003.

2. Instituto Especializado de Salud Mental Honorio Delgado - Hideyo Noguchi. Estudio Epidemiológico de Salud Mental en la Sierra Peruana 2003. Informe General. Anales de Salud Mental 2004; 19 (1-2): 1-216.

3. Organización Mundial de la Salud. Informe sobre la Salud en el Mundo 2002. Ginebra: Organización Mundial de la Salud. URL disponible en: http://www.who.int/ whr/2002/es/whr02_es.pdf (Fecha de acceso: marzo del 2005).

4. Hemenway D, Solnick SJ. The unintentional injurer: results from the Boston youth survey. Am J Public Health 2011;101(4):663-8.

5. Cherpitel CJ. Alcohol and injuries: a review of international emergency room studies. Addiction 1993; 88: 923-937.

6. El-Guebaly N, Armstrong S, Hodgins DC. Substance abuse and the emergency room: programmatic implications. J Addict Dis 1998; 17(2), 21-40.

7. Cherpitel CJ, Bond J, Ye Y, Borges G, Macdonald S, Giesbrecht N. A cross-national metaanalysis of alcohol and injury: data from the Emergency Room Collaborative Alcohol Analysis Project (ERCAAP). Addiction 2003; 98(9): 1277-1286.

8. Cherpitel CJ, Ye Y, Bond J. Alcohol and injury: multilevel analysis from the Emergency Room Collaborative Alcohol Analysis Project (ERCAAP). Alcohol Alcohol 2004; 39: 552-558.

9. Rockett IR, Putnam SL, Jia H, Chang CF, Smith GS. Unmet substance abuse treatment need, health services utilization, and cost: a population-based emergency department study. Ann Emerg Med 2005; 45:118-27.

10. Substance Abuse and Mental Health Services Administration, Office of Applied Studies. Drug Abuse Warning Network, 2007: National Estimates of DrugRelated Emergency Department Visits. Rockville, MD: U.S. Department of Health and Human Services; 2010. 
11. Bejarano J, Sáenz M. Trauma y consumo de drogas en salas de emergencia en hospitales generales costarricences, 1998-2002. Revista Peruana de Drogodependencias 2005; 3(1): 129-144.

12. Perales A, Sogi C, García M, Flores R, Gonzáles D, Torres $\mathrm{R}$, et al. Sistema de Registro del Uso y Abuso de Alcohol y Drogas (RENAD). Lima: MINSA; 1990.

13. Vitale SG, Van De Mheen D, Van De Wiel A, Garretsen HF. Alcohol and illicit drug use among emergency room patients in the Netherlands. Alcohol Alcohol 2006; 41 (5): 553-559.

14. Mazzotti G, Ordoñez C, Bustamante I, Acostas R, Contreras C, Gallo C, Fiestas F. Consumo de alcohol y otras sustancias psicoactivas en pacientes atendidos en un servicio de emergencia en Lima-Perú. Revista Peruana de Drogodependencias 2006; 4(1): 115-135.
15. D’Onofrio G, Degutis L. Integrating Project ASSERT: A Screening, Intervention, and Referral to Treatment Program for Unhealthy Alcohol and Drug Use Into an Urban Emergency Department. Acad Emerg Med 2010; 17:903-911.

16. Fiestas F, Ponce J, Gallo C, Bustamante I, Ordóñez C, Mazzotti G. Factores predictores de uso problemático de alcohol en personas atendidas en una sala de emergencia. Rev Peru Med Exp Salud Publica 2011;28 (1):54-61.

17. Madras BK, Compton WM, Avula D, Stegbauer T, Stein JB, Clark HW. Screening, brief interventions, referral to treatment (SBIRT) for illicit drug and alcohol use at multiple healthcare sites: comparison at intake and 6 months later. Drug Alcohol Depend 2009; 99(1-3): 28095. 